\title{
IPA Liver Cancer Regimen
}

National Cancer Institute

\section{Source}

National Cancer Institute. IPA Liver Cancer Regimen. NCI Thesaurus. Code C67296.

A regimen consisting of ifosfamide, cisplatin and doxorubicin, used in the neoadjuvant setting for the treatment of advanced-stage, childhood hepatoblastoma. 